\title{
Rapidly deteriorating course in Dutch hereditary spastic paraplegia type 11 patients
}

\author{
Susanne T de Bot ${ }^{1}$, Rogier C Burggraaff ${ }^{2}$, Johanna C Herkert ${ }^{2}$, Helenius J Schelhaas ${ }^{1}$, Bart Post $^{1}$, \\ Adinda Diekstra ${ }^{3}$, Reinout O van Vliet ${ }^{4}$, Marjo S van der Knaap ${ }^{5}$, Erik-Jan Kamsteeg ${ }^{3}$, Hans Scheffer ${ }^{3}$, \\ Bart P van de Warrenburg ${ }^{1}$, Corien C Verschuuren-Bemelmans ${ }^{2}$ and Hubertus PH Kremer ${ }^{\star, 6}$
}

\begin{abstract}
Although SPG11 is the most common complicated hereditary spastic paraplegia, our knowledge of the long-term prognosis and life expectancy is limited. We therefore studied the disease course of all patients with a proven SPG11 mutation as tested in our laboratory, the single Dutch laboratory providing SPG11 mutation analysis, between 1 January 2009 and 1 January 2011. We identified nine different SPG11 mutations, four of which are novel, in nine index patients. Eighteen SPG11 patients from these nine families were studied by means of a retrospective chart analysis and additional interview/examination. Ages at onset were between 4 months and 14 years; 39\% started with learning difficulties rather than gait impairment. Brain magnetic resonance imaging showed a thin corpus callosum and typical periventricular white matter changes in the frontal horn region (known as the 'ears-of the lynx'-sign) in all. Most patients became wheelchair bound after a disease duration of 1 to 2 decades. End-stage disease consisted of loss of spontaneous speech, severe dysphagia, spastic tetraplegia with peripheral nerve involvement and contractures. Several patients died of complications between ages 30 and 48 years, 3-4 decades after onset of gait impairment. Other relevant features during the disease were urinary and fecal incontinence, obesity and psychosis. Our study of 18 Dutch SPG11-patients shows the potential serious long-term consequences of SPG11 including a possibly restricted life span.
\end{abstract}

European Journal of Human Genetics (2013) 21, 1312-1315; doi:10.1038/ejhg.2013.27; published online 27 February 2013

Keywords: corpus callosum/pathology; mutation/genetics; prognosis; psychoses; hereditary spastic paraplegia; human SPG11 protein

\section{INTRODUCTION}

Hereditary spastic paraplegias (HSPs) are a clinically and genetically heterogeneous group of neurodegenerative disorders. In addition to pure forms, in which symptoms are limited to lower limb pyramidal tract dysfunction, complicated forms exist with additional neurological signs and usually an autosomal recessive (AR) pattern of inheritance.

SPG11, caused by mutations in the SPG11 gene encoding the protein spatacsin in which currently $>120$ mutations have been described, is the most frequent AR-HSP worldwide, accounting for about $40 \%$ of complex AR-HSPs. ${ }^{1}$ SPG11 is characterized by earlyonset spastic paraplegia and cognitive deficits with variable other signs, like peripheral neuropathy, hand muscle atrophy and cerebellar ataxia. Brain magnetic resonance imaging (MRI) shows nonspecific cerebral atrophy, a characteristically thin (anterior) corpus callosum and periventricular white matter changes (WMCs) in the frontal horn region, with a typical 'ears of the lynx'-appearance. ${ }^{2}$ Other clinical syndromes have also been reported as being associated with SPG11 mutations, for example, Kjellin syndrome, juvenile amyotrophic lateral sclerosis and L-dopa-responsive parkinsonism. ${ }^{3-5}$

Onset of SPG11 usually occurs within the second decade of life and the disease progresses relatively rapidly as compared with other HSPs. ${ }^{6}$ Most patients become wheelchair bound after 1 or 2 decades.
Little information is available about the long-term prognosis and life expectancy. This prompted us to study the disease course of SPG11 patients in the Netherlands.

\section{MATERIALS AND METHODS}

Patients

Since 2009, the DNA diagnostic laboratory of the Radboud University Nijmegen Medical Center is the only Dutch laboratory providing SPG11 mutation analysis. From January 2009 till January 2011, mutation analysis for SPG11 was performed in 125 spastic paraplegia patients of whom DNA samples and clinical data were submitted by various clinicians from all over the country. All patients or their caregivers had provided written informed consent. These 125 patients presented a broad phenotypical spectrum, with or without additional signs or abnormalities on brain MRI. For all patients with a proven SPG11 mutation, available medical records and imaging data were reviewed. A clinicogenetic HSP database was constructed containing clinical information about the age at onset of gait impairment and learning difficulties (as mentioned by the patient or parents), symptoms, neurological examination, and the results of genetic testing and brain MRI. A neurological re-examination and/or an additional (phone) interview with the relatives has been performed focused on deficient clinical data (all patients in Supplementary Table 1 except for patient 7 who was lost to follow-up). This study has been carried out in the Netherlands in accordance with the rules set by the national research ethics committees on the review of studies.

${ }^{1}$ Department of Neurology, Radboud University, Nijmegen Medical Centre, Donders Institute for Brain, Cognition and Behaviour, Nijmegen, The Netherlands; ${ }^{2}$ Department of Genetics, University Medical Center Groningen, University of Groningen, Groningen, The Netherlands; ${ }^{3}$ Department of Human Genetics, Radboud University, Nijmegen Medical Centre, Donders Institute for Brain, Cognition and Behaviour, Nijmegen, The Netherlands; ${ }^{4}$ Roessingh, Centre of Rehabilitation, Enschede, The Netherlands; ${ }^{5}$ Department of Child Neurology, VU University Medical Centre, Amsterdam, The Netherlands; ${ }^{6}$ Department of Neurology, University Medical Center Groningen, University of Groningen, Groningen, The Netherlands

${ }^{*}$ Correspondence: Professor Dr HPH Kremer, Department of Neurology - AB51, University Medical Center Groningen, University of Groningen, PO Box 30 001, 9700 RB Groningen, The Netherlands. Tel: +31 050 3612430; E-mail: h.p.h.kremer@umcg.nl

Received 27 September 2012; revised 23 December 2012; accepted 22 January 2013; published online 27 February 2013 


\section{Genetic analysis}

Mutation analysis of the SPG11 gene was performed by standard Sanger sequencing of the coding sequences, including flanking intronic regions, and MLPA analysis (MRC-Holland kit P-306, Amsterdam, The Netherlands). Genbank Accession Number NM_025137.3 was used as the reference sequence. Mutation nomenclature follows the guidelines from the Human Genome Variation Society (http://www.hgvs.org/mutnomen/), version 2.

\section{RESULTS}

\section{Genotype}

In a sample of 125 (pure and complex) HSP patients, we identified nine different SPG11 mutations in nine kindreds (7.2\%; Table 1). Four mutations were novel, and all were predicted to be null mutations, that is, nonsense, splice site or frame-shift mutations, causing a premature termination codon resulting in absence of the encoded spatacsin protein.

One particular mutation, a deletion of exons 31-34 (c.5867?.6477+ ?del), was identified in five index patients. Extensive genealogical analysis identified a common ancestor for patients 6, 9 and both patients of family 3 .

\section{Phenotype}

The 18 SPG11 patients from nine families were examined at a mean age of 26 years (range 13-46 years). All had a prominent spastic paraplegia with cognitive deficits (Supplementary Table 1). The disease presented with either motor/gait impairment (11 patients, $61 \%$ ) or learning difficulties (7 patients, 39\%) between the ages of 4 months and 14 years (mean 7.9 years).

At the time of examination, after a mean of 15.9 years (range 0.9 35.3 years) since the occurrence of gait impairment, 12 patients $(67 \%)$ were no longer ambulatory. They had become wheelchair bound after 2-18 years of gait impairment. During follow-up, 94\% developed sphincter problems, manifesting as urinary as well as fecal incontinence in $44 \%$. Obesity was seen in 14 patients in total $(78 \%)$, of whom 9 were non-ambulatory. From the four patients who were not obese, three were wheelchair bound for $>15$ years. At least, 7 out of 13 patients (54\%) developed dysphagia, necessitating tube feeding in 3 of them. Cerebellar ataxia was seen in 8 out of $14(57 \%)$ patients. No prominent extrapyramidal signs were noted. Four patients $(22 \%)$ from three separate families suffered from one or more episodes of psychosis at ages 24 to 28 years. Another five patients had behavioral problems in childhood.

Brain MRIs from nine patients were available for review. Both a thin corpus callosum and periventricular WMCs in the frontal horn region were seen in all nine patients; this last feature fitted the description of the 'ears-of the lynx'-sign (Figure 1). In seven patients, cerebral atrophy was noted as well.

\section{Long-term follow-up and end-stage SPG11-HSP}

After $>20$ disease years, striking features of the end-stage condition were: wheelchair-bound tetraplegia with flexion contractures, severe amyotrophy of the hand muscles, urinary and fecal incontinence, dysphagia, dementia and finally mutism (see also Supplementary Material: Table S1, Figure S1 and family report). Five patients died of complications at a mean age of 41.6 years (range: $30-48$ years); 26 to 47 years after disease onset.

\section{DISCUSSION}

Within the spectrum of HSPs, SPG11 can present not only as an almost 'uncomplicated' HSP, but can also evolve into a severe disorder with progressive invalidating features, like in a substantial proportion of our patients, leading to complete dependency or death before the age of 50. Thirty-nine percent of our patients started with isolated learning difficulties. As gait impairment raises the suspicion of an HSP earlier, learning difficulties as the presenting symptom might have been under-represented in previous studies. $1,7,8$

Most patients lost ambulation 1 to 2 decades after disease onset, regardless of supportive therapeutic strategies. The percentage of wheelchair-bound subjects was 67\% after 15.9 years (mean) of gait impairment, which is only slightly higher than reported previously. ${ }^{1}$ The typical brain MRI signs described before could also be confirmed. ${ }^{1,2}$

The speed of disease progression resulted in a limited lifespan of 3 to 4 decades after disease-onset. In other reports progression to nonambulation varied from 1 or 2 decades to intact ambulation after 24 years. $^{6,9}$ Our study underscores the potential serious long-term consequences of SPG11 including a restricted life span.

A comparably rapid progression with predominant learning difficulties at onset, followed by severe spastic paraplegia with distal amyotrophy, hallucinations in adolescence, aphasia, obesity, and fecal

Table 1 Mutations identified in the SPG11 gene in nine kindreds

\begin{tabular}{|c|c|c|c|c|c|c|c|c|}
\hline Kindred & No. aff. & Zygosity & Origin & Ex/intr & Mutation (cDNA) & Amino-acid subst. & Type & Reference \\
\hline 1 & 5 & Homoz. & TU & Ex 6 & c.1203 del & p. (Asp402fs) & Frameshift & Stevanin et al ${ }^{p}$ \\
\hline \multirow[t]{2}{*}{2} & \multirow[t]{2}{*}{3} & \multirow[t]{2}{*}{ Heteroz. } & \multirow[t]{2}{*}{ NL } & Ex 28 & c.4811dup & p. (Leu 1605fs) & Frameshift & This report \\
\hline & & & & Ex 36 & c.6739_6742del & p. (Glu2247fs) & Frameshift & Stevanin et al ${ }^{1}$ \\
\hline \multirow[t]{2}{*}{3} & \multirow[t]{2}{*}{2} & \multirow[t]{2}{*}{ Heteroz. } & \multirow[t]{2}{*}{ NL } & Ex 6 & c.1348dup & p.(Ile450fs) & Frameshift & Paisan-Ruiz (2008) Neurology 70: 1384 \\
\hline & & & & Ex 31-34 & c.5867-?_6477+?del & p.(?) & Exon deletion & Denora et $a^{\beta a}$ \\
\hline \multirow[t]{2}{*}{4} & \multirow[t]{2}{*}{4} & \multirow[t]{2}{*}{ Heteroz. } & \multirow[t]{2}{*}{$\mathrm{NL}$} & Ex 6 & c. $1085 \mathrm{G}>\mathrm{A}$ & p. $\left(\operatorname{Trp} 362^{*}\right)$ & Nonsense & This report \\
\hline & & & & Ex 6 & c.1348dup & p.(Ile450fs) & Frameshift & Paisan-Ruiz (2008) Neurology 70: 1384 \\
\hline \multirow[t]{2}{*}{5} & \multirow[t]{2}{*}{1} & \multirow[t]{2}{*}{ Heteroz. } & \multirow[t]{2}{*}{ NL } & Ex 30 & c. $5623 \mathrm{C}>\mathrm{T}$ & p. $\left(\mathrm{G} \ln 1875^{*}\right)$ & Nonsense & Paisan-Ruiz (2008) Eur J Neurol 15: 1065 \\
\hline & & & & Ex 31-34 & c.5867-?_6477+?del & p.(?) & Exon deletion & Denora et $a^{\beta a}$ \\
\hline 6 & 1 & Homoz. & NL & E 31-34 & c.5867-?_6477+?del & p.(?) & Exon deletion & Denora et $a^{\beta a}$ \\
\hline 7 & 1 & Homoz. & so & Ex 36 & c.6709del & p. (Ala2237fs) & Frameshift & This report \\
\hline \multirow[t]{2}{*}{8} & \multirow[t]{2}{*}{1} & \multirow[t]{2}{*}{ Heteroz. } & \multirow[t]{2}{*}{ NL } & Intron 32 & c. $6205+1 \mathrm{del}$ & p.(?) & Donor splice site & This report \\
\hline & & & & Ex 31-34 & c.5867-?_6477+?del & p.(?) & Exon deletion & Denora et $a \beta a$ \\
\hline \multirow[t]{2}{*}{9} & \multirow[t]{2}{*}{1} & \multirow[t]{2}{*}{ Heteroz. } & \multirow[t]{2}{*}{ NL } & Ex 6 & c.1348dup & p.(Ile450fs) & Frameshift & Paisan-Ruiz (2008) Neurology 70: 1384 \\
\hline & & & & Ex 31-34 & c.5867-?_6477+?del & p.(?) & Exon deletion & Denora et $a{ }^{\beta a}$ \\
\hline
\end{tabular}

Abbreviations: No. aff, number of affected individuals; Homoz., homozygous; Heteroz., heterozygous; Ex/intr, exon/intron; NL, The Netherlands; SO, Somalia; TU, Turkey.

apreviously described as: c.5898 + 5493_6509-491del, we have not determined the break points. Bold mutations are novel. 

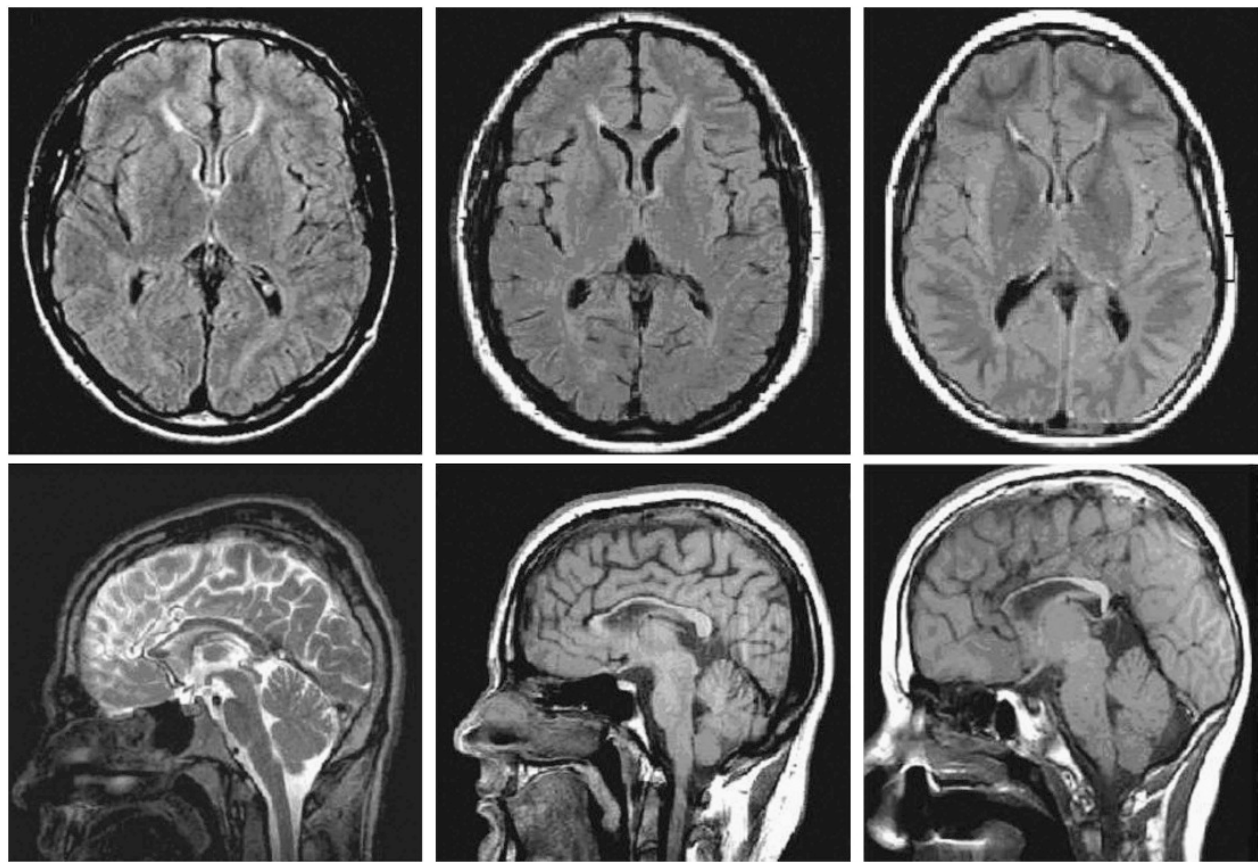

Figure 1 Brain MRI of three SPG11 patients (patient 3-V-2, 5 and 9 on the right; Supplementary Table 1). Top: axial FLAIR T2 images showing periventricular WMCs in the typical 'flame- or 'ears-of the lynx' formation'; and below: saggital T1 and T2 images showing a thin corpus callosum, especially the anterior part.
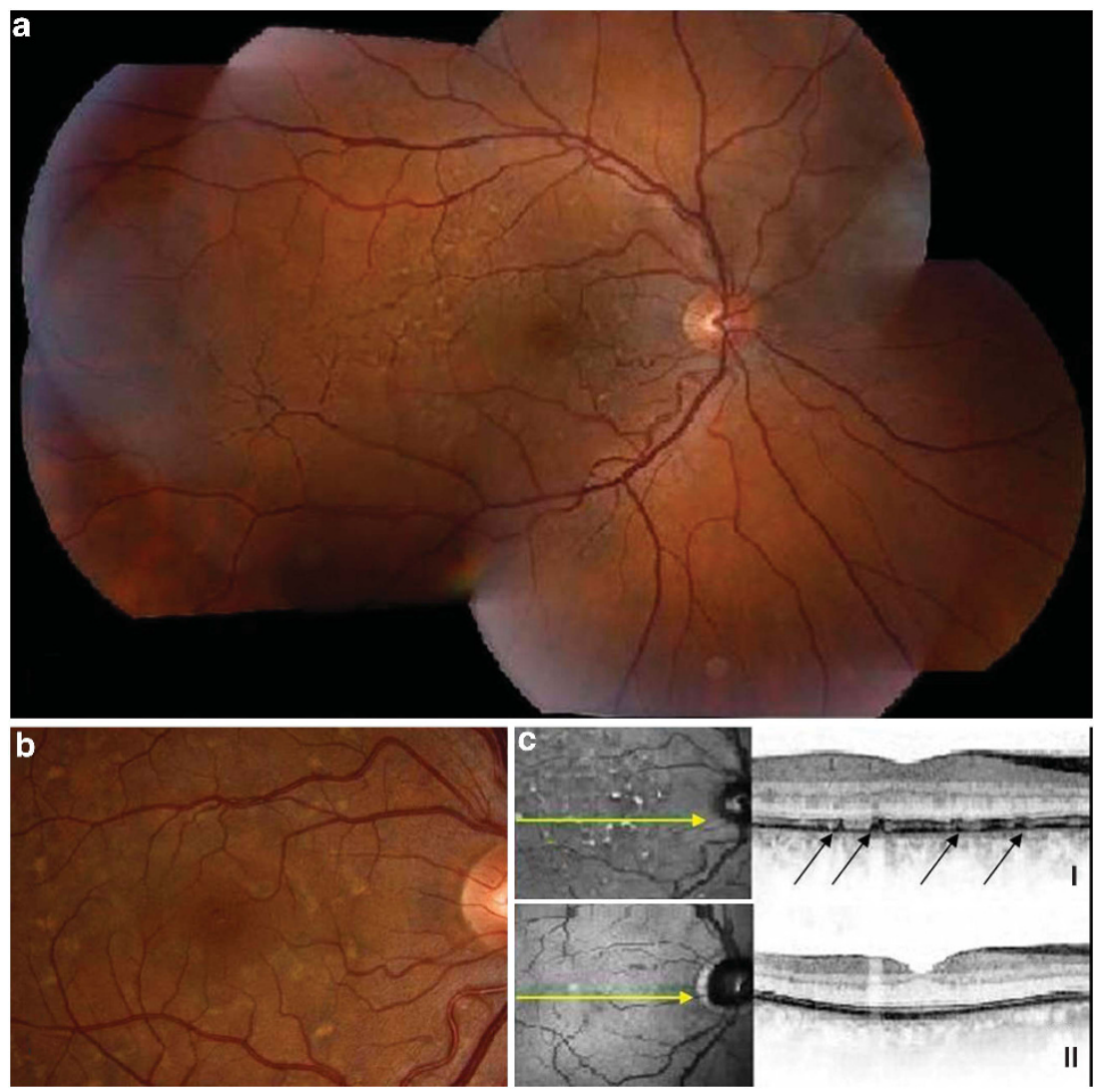

Figure 2 Fundus photographs of patient 4-II-3 (Supplementary Table 1) showing retinal pigment epithelium lesions seen as multiple pale, yellow spots and dark scattered pigmentations after 19 years of disease duration; (a): combined overview of right retina; (b): details in posterior pole; (c): using optical coherence tomography I: the lesions (arrows) proved to be at the level of the retinal pigment epithelium, compared with II: normal retinal pigment epithelium in a healthy person. 
and urinary incontinence, was recently described in seven patients with Kjellin syndrome (four of whom had SPG11 mutations). ${ }^{10}$ This condition is also characterized by typical retinal pigment epithelium lesions later in the disease. ${ }^{10}$ Two of these patients died at ages 30 and 36 because of pulmonary complications. Whether this aggressive disease course is specifically associated with Kjellin syndrome is questionable, since in its first description, SPG11 mutations in five patients indicated a much milder course with a relatively late onset of cognitive decline in the fourth decade. ${ }^{5}$ In our SPG11 cohort, two (twin brothers) out of six patients, of whom ophthalmological data were available, had retinal pigment epithelium lesions after 12 to 19 years of disease, compatible with Kjellin syndrome (Figure 2). Unfortunately, ophthalmologic examination was performed in six patients only.

The strikingly reduced verbal fluency leading to mutism seen in some of our patients (families 1 and 2; Supplementary Table 1) has not been described in SPG11 patients with Kjellin syndrome, but has been reported in two members of an Italian SPG11 family. ${ }^{11}$ Obesity — not because of immobility—was a remarkable frequent feature, as well as episodes of psychosis. Both symptoms have been described in some SPG11 patients, but have not been considered part of the phenotype before. ${ }^{1,5,8,10,12}$ Finally, we found a much higher percentage of sphincter disturbances than previous studies $(94 \%$ vs $42.9 \%)$, and urinary and fecal incontinence in particular (44\% vs unknown), possibly due to under-reporting before. ${ }^{7}$ Whether obesity, hallucinations and muscle atrophy are part of the SPG11 phenotype itself or are actually secondary to immobility, comorbidity or comedication, needs further attention in future studies, as well as the possibility of a more severe disease course in patients with retinal pigment epithelium lesions (Kjellin syndrome).

The largely retrospective nature of our study imposes limitations. Nevertheless, our study contributes to the knowledge about the clinical phenotype of SPG11, including notable clinical signs and symptoms like mutism, psychosis, dysphagia, obesity and sphincter disturbances in the course of the disease, as well as typical neuroimaging signs. Moreover, our study underscores the more rapidly deteriorating course as compared with other HSPs and a possible restricted life span because of complications in some SPG11 patients. This may aid in raising diagnostic awareness and in improving case management, prognosis and counseling of subjects and their families with this complex multisystem disease.

\section{CONFLICT OF INTEREST}

The authors declare no conflict of interest.

\section{ACKNOWLEDGEMENTS}

We thank all the patients and their families. We also thank Dr Gruijters for providing additional clinical details, Dr E Veldman and the medical photographers of the Medical Center in Enschede for providing the fundus photographs and OCT images, and Jackie Senior for editing the text. This work was supported by the European Community's Seventh Framework Program FP7/2007-2013 under grant agreement n ${ }^{\circ} 223143$ (project acronym: TECHGENE) to Dr Scheffer. This funding body as well as the following ones had no role in the design of the study, collection and analysis of data and decision to publish. Dr van de Warrenburg receives or has received research support from the Prinses Beatrix Fonds, the European Union, the Brain Foundation, Ipsen Pharmaceuticals, the Grossweiler Foundation and the Royal Dutch Society for Physical Therapy. Professor Kremer receives or has received research support from the University Medical Center Groningen, and the BCN-Brain Research Institute. This study was not industry-sponsored.

\section{AUTHOR CONTRIBUTIONS}

SdB: study concept and design, acquisition of data, analysis and interpretation and final revisions. RCB, JCH, HJS, BP, AD, ROvV and MSvdK: acquisition of data, and critical revision of the manuscript for important intellectual content. BPvdW and HS performed critical revision of the manuscript for important intellectual content. HPHK and CCV-B: study concept and design, acquisition of data, critical revision of the manuscript for important intellectual content, study supervision.

1 Stevanin G, Azzedine H, Denora P et al: Mutations in SPG11 are frequent in autosomal recessive spastic paraplegia with thin corpus callosum, cognitive decline and lower motor neuron degeneration. Brain 2008; 131: 772-784.

2 Riverol M, Samaranch L, Pascual B et al: Forceps minor region signal abnormality 'ears of the Iynx': an early MRI finding in spastic paraparesis with thin corpus callosum and mutations in the spatacsin gene (SPG11) on chromosome 15. J Neuroimaging 2009; 19: 52-60.

3 Anheim M, Lagier-Tourenne C, Stevanin G et al: SPG11 spastic paraplegia. A new cause of juvenile parkinsonism. J Neurol 2009; 256: 104-108.

4 Orlacchio A, Babalini C, Borreca A et al: SPATACSIN mutations cause autosomal recessive juvenile amyotrophic lateral sclerosis. Brain 2010; 133: 591-598.

5 Orlen H, Melberg A, Raininko R et al: SPG11 mutations cause Kjellin syndrome, a hereditary spastic paraplegia with thin corpus callosum and central retinal degeneration. Am J Med Genet B Neuropsychiatr Genet 2009; 150B: 984-992.

6 Hehr U, Bauer P, Winner B et al: Long-term course and mutational spectrum of spatacsin-linked spastic paraplegia. Ann Neurol 2007; 62: 656-665.

7 Stevanin G, Santorelli FM, Azzedine H et al: Mutations in SPG11, encoding spatacsin, are a major cause of spastic paraplegia with thin corpus callosum. Nat Genet 2007; 39: 366-372.

8 Denora PS, Schlesinger D, Casali C et al: Screening of ARHSP-TCC patients expands the spectrum of SPG11 mutations and includes a large scale gene deletion. Hum Mutat 2009; 30: E500-E519.

9 Erichsen AK, Stevanin G, Denora P et al: SPG11-the most common type of recessive spastic paraplegia in Norway? Acta Neurol Scand Suppl 2008; 188: 46-50.

10 Puech B, Lacour A, Stevanin G et al: Kjellin syndrome: long-term neuro-ophthalmologic follow-up and novel mutations in the SPG11 gene. Ophthalmology 2011; 118: 564-573.

11 Del Bo, Di FA, Ghezzi S et al: SPG11: a consistent clinical phenotype in a family with homozygous spatacsin truncating mutation. Neurogenetics 2007; 8: 301-305.

12 Lossos A, Stevanin G, Meiner V et al: Hereditary spastic paraplegia with thin corpus callosum: reduction of the SPG11 interval and evidence for further genetic heterogeneity. Arch Neurol 2006; 63: 756-760.

Supplementary Information accompanies this paper on European Journal of Human Genetics website (http://www.nature.com/ejhg) 